\title{
Nanoparticle Embedded Double-Layer Coating for Daytime Radiative Cooling
}

\author{
Zhifeng Huang, ${ }^{\mathrm{a}, \mathrm{b}}$ Xiulin Ruan, ${ }^{\mathrm{a} *}$ \\ ${ }^{a}$ School of Mechanical Engineering and the Birck Nanotechnology Center, Purdue University, West \\ Lafayette, Indiana 47907, USA \\ ${ }^{\mathrm{b}}$ School of Power and Mechanical Engineering, Wuhan University, Wuhan, Hubei 430072, China
}

\begin{abstract}
Radiative cooling is a passive cooling method by emitting heat to outer space without energy input. In this work we propose a cost effective double-layer coating embedded with nanoparticles for both nighttime and daytime radiative cooling. The top and bottom layers are acrylic resin embedded with titanium dioxide and carbon black particles, respectively responsible for reflecting the solar irradiation and emitting the heat in the atmospheric transparency window. The carbon black layer is considered as the black substrate. For the top layer, different sizes of titanium dioxide particles are examined, and $0.2 \mu \mathrm{m}$ radius is found to give the best cooling performance. More than $90 \%$ of the solar irradiation can be reflected, and the average emissivity in the atmospheric transparency window is larger than 0.9 in most directions. A daytime net cooling power over 100 $\mathrm{W} / \mathrm{m}^{2}$ is predicted at the ambient temperature. The cooling effect persists even if significant conduction and convection heat exchange is considered.
\end{abstract}

Keywords: Radiative cooling; Particle embedded coating; Monte Carlo simulation; Thermal radiation; Selective radiative property

\footnotetext{
* Corresponding author: ruan@purdue.edu
} 


\section{INTRODUCTION}

Cooling represents a significant sector of energy consumption. Radiative cooling, as a passive cooling method, can cool a structure without energy input. It utilizes the fact that the atmosphere is nearly transparent in the "sky window" between 8-13 $\mu \mathrm{m}$, which allows for the emission of thermal radiation in this range from the surface to outer space.

Radiative cooling has been investigated for a long time, and many previous works have achieved nighttime cooling. However, the largest cooling power demand usually occurs at daytime under the direct sunlight, and the coolers need to have both high emissivity in the "sky window" and high reflectivity in the solar spectrum, which are difficult to achieve simultaneously. Several methods have been used to achieve night-time or day-time cooling effects. The first approach is to use materials of selective radiative properties. Selectively infrared emitting gases, such as ammonia $\left(\mathrm{NH}_{3}\right)$, ethylene $\left(\mathrm{C}_{2} \mathrm{H}_{4}\right)$, and ethylene oxide $\left(\mathrm{C}_{2} \mathrm{H}_{4} \mathrm{O}\right)$, which have high emissivity in the "sky window", are used for radiative cooling [1-2]. Some other materials including polyvinyl-fluoride plastic film and silicon monoxide film on aluminum also show good performance as selective emitters [3-4]. The second approach is to use periodic nanostructures [5-9] including photonic crystals and metamaterials due to the advancements of fabrication technologies in recent years. A daytime radiative cooler was proposed to have a good cooling performance by numerical simulation [8]. It consists of two thermally emitting photonic crystal layers, below which lies a broadband solar reflector. An integrated photonic solar reflector and thermal emitter were fabricated and experimentally demonstrated for radiative cooling under direct sunlight for the first time by the same group [9]. However, the nanostructures used in 
these works are complicated and expensive, which will limit their large scale applications. The third approach, which is more scalable, is to embed micro/nano-particles in a matrix to form a coating or cover of selective radiative properties [10-17]. In some works [11-15], a cover foil made of particle embedded polyethylene was designed to have high reflectivity in the solar spectrum and high transparency in the "sky window". These two requirements are usually in conflict with each other, requiring some compromise in the design. Meanwhile, in order to obtain good cooling performance, the emitter underneath needs to have high emissivity in the "sky window". Some other works [16-17] have focused on both the thermal and esthetic performance that require high absorptivity in the visible range, limiting their performances for daytime cooling. A cost effective and scalable radiative cooler design for daytime radiative cooling still needs further exploration.

In this work, we propose a cost effective double-layer coating embedded with particles for both nighttime and daytime radiative cooling. The two layers are acrylic resin embedded with titanium dioxide and carbon black particles, respectively responsible for reflecting the solar irradiation and emitting the heat in the "sky window". In this way, regardless of how small the emissivity of the original surface in the "sky window" is, daytime cooling can be achieved after coating with these two layers, which is superior to previous cover designs [11-15]. The spectral radiative properties of the double-layer coating are calculated numerically, considering the effects of the particle size, particle concentration, and the coating thickness. Then, the cooling power is analyzed at both daytime and nighttime. 


\section{THEORY AND MODELING METHODS}

Selective radiative properties of the particle embedded coating are crucial for the radiative cooling application. The direct method to calculate the spectral radiative properties is to solve the Maxwell's equations, since the particle size is comparable to the incident wavelength and the wave effect should be considered. However, this calculation is too time-consuming to be practical for our system. Here we seek an alternative way. First, the electromagnetic wave theory is used to calculate the radiative properties of a single particle. Assume the scattering behavior of each particle embedded in the coating is independent of the others for the low particle volume fraction considered in this work, the radiative properties of the particle embedded coating including absorption coefficient, scattering coefficient, and scattering phase function are readily calculated. Then, the obtained radiative properties are used to solve the Radiative Transfer Equation (RTE) to calculate the spectral reflectivity and emissivity of the coating. This approach has a good accuracy and is much more efficient than solving the Maxwell's equations directly [16-17]. Finally, the cooling power of the coating under different conditions is evaluated.

\subsection{Materials Selection}

As shown in Fig. 1, our coating has a double-layer structure for daytime radiative cooling. The bottom layer designed to have high emissivity in the "sky window" in order to output heat from the surface. The top layer is used to reflect solar energy and needs to have high reflectivity in the solar spectrum. At the same time, it needs to have low reflectivity in the "sky window" in order not to 
reflect back the infrared emission by the bottom layer.

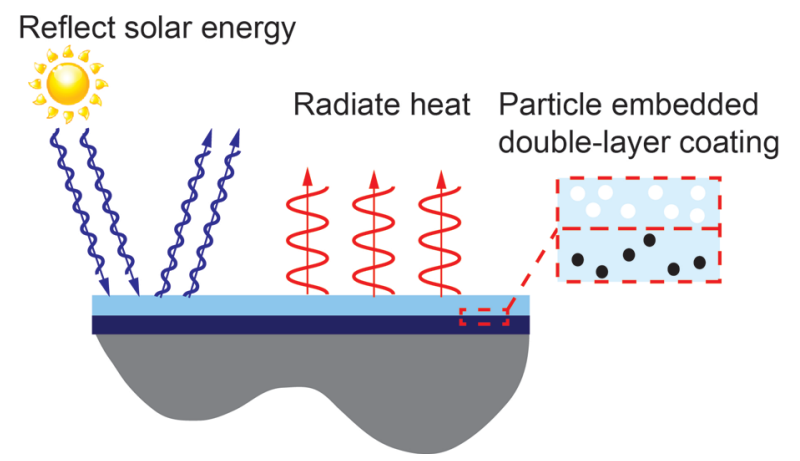

Figure 1 Sketch of a functional double-layer coating used for daytime radiative cooling

In order to achieve high reflectivity in the solar spectrum, the particle material needs to have a large bandgap so the solar irradiation will not be absorbed; meanwhile, the particle material needs to have a high refractive index. If the particle size is properly chosen, high reflectivity in the solar spectrum and low reflectivity in the "sky window" can be obtained simultaneously, since the particle size that effectively reflects solar irradiation is usually much smaller than the long wavelength of the "sky window" radiation, hence the particles do not significantly scatter photons in this range. Titanium dioxide of rutile phase is not absorptive in most of the solar spectrum and has high refractive index in the spectrum [18]. It is chosen as the particle material for the top layer. The particles are embedded in acrylic resin because of the high transparency of acrylic resin in the whole considered spectrum range.

For the bottom layer, the only requirement is high emissivity in the "sky window". Carbon black is a good choice for its high absorptivity and abundancy. It behaves like a blackbody in absorbing radiative energy [19]. The carbon black particles can be either embedded in the acrylic resin or simply sprayed on the bottom surface of the top layer to form the second layer, which is used for emitting heat in the "sky window". For simplicity it is considered as a black boundary of the top layer in this 
work.

\subsection{Radiative Properties of Single Particles}

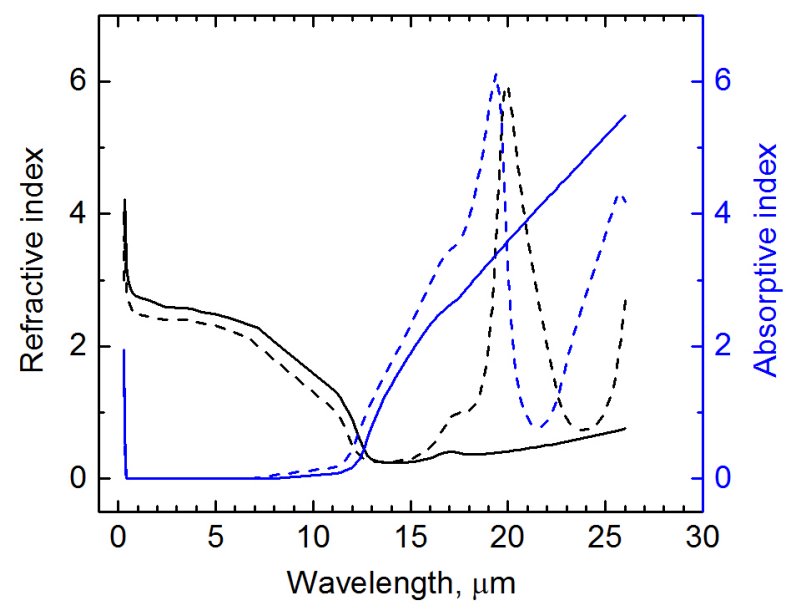

Figure 2 Complex index of refraction of $\mathrm{TiO}_{2}$ in the rutile state (Solid: Parallel polarization; Dash:

\section{Perpendicular polarization)}

The radiative properties of a single spherical particle of radius $r$, interacting with an electromagnetic wave of wavelength $\lambda$, are governed by two independent nondimensional parameters: complex index of refraction $m=n-i k$, and size parameter $x=2 \pi r / \lambda$. Figure 2 shows the spectral complex index of refraction of $\mathrm{TiO}_{2}$ of rutile crystal structure in the range of $0.3-26 \mu \mathrm{m}$ [18]. Since the structure cannot be treated as a cubic crystal, there are pronounced polarization effects. Therefore, two spectral optical constants which are respectively corresponding to the parallel and perpendicular polarization, referring to the radiation electrical filed parallel and perpendicular to the $c$ axis of the crystal, are given in Fig. 2. In this work, since the particles are embedded in an acrylic resin with a refractive index $n_{0}=1.5$, the complex index of refraction for the particles used in the following 
calculations should be changed from $(n-i k)$ to $(n-i k) / n_{0}$.

Once the complex index of refraction and the size of the particle is known, the radiative properties, usually represented by the extinction efficiency factor $Q_{e x t}$, scattering efficiency factor $Q_{s c a}$, and scattering phase function $\Phi(\Theta)$, can be calculated by solving the Maxwell's equations [20]

$$
\begin{gathered}
Q_{\text {ext }}=\frac{2}{x^{2}} \sum_{n=1}^{\infty}(2 n+1) \Re\left\{a_{n}+b_{n}\right\} \\
Q_{\text {sca }}=\frac{2}{x^{2}} \sum_{n=1}^{\infty}(2 n+1)\left(\left|a_{n}\right|^{2}+\left|b_{n}\right|^{2}\right) \\
\Phi(\Theta)=2 \frac{i_{1}+i_{2}}{x^{2} Q_{s c a}}
\end{gathered}
$$

The Mie scattering coefficients $a_{n}$ and $b_{n}$ are complex functions of $x$ and $y=m x$,

$$
\begin{aligned}
& a_{n}=\frac{\psi_{n}^{\prime}(y) \psi_{n}(x)-m \psi_{n}(y) \psi_{n}^{\prime}(x)}{\psi_{n}^{\prime}(y) \zeta_{n}(x)-m \psi_{n}(y) \zeta_{n}^{\prime}(x)} \\
& b_{n}=\frac{m \psi_{n}^{\prime}(y) \psi_{n}(x)-\psi_{n}(y) \psi_{n}^{\prime}(x)}{m \psi_{n}^{\prime}(y) \zeta_{n}(x)-\psi_{n}(y) \zeta_{n}^{\prime}(x)}
\end{aligned}
$$

where the function $\psi_{n}$ and $\zeta_{n}$ are known as Riccati-Bessel functions.

The nondimensional polarized intensities $i_{1}$ and $i_{2}$ in Eq. (3) are calculated from

$$
\begin{gathered}
i_{1}(x, m, \Theta)=\left|S_{1}\right|^{2}, \quad i_{2}(x, m, \Theta)=\left|S_{2}\right|^{2} \\
S_{1}(\Theta)=\sum_{n=1}^{\infty} \frac{(2 n+1)}{n(n+1)}\left[a_{n} \pi_{n}(\cos \Theta)+b_{n} \tau_{n}(\cos \Theta)\right] \\
S_{2}(\Theta)=\sum_{n=1}^{\infty} \frac{(2 n+1)}{n(n+1)}\left[b_{n} \pi_{n}(\cos \Theta)+a_{n} \tau_{n}(\cos \Theta)\right]
\end{gathered}
$$

where the directional dependent functions $\pi_{n}$ and $\tau_{n}$ are related to Legendre polynomials $P_{n}$ by

$$
\pi_{n}(\cos \Theta)=\frac{d P_{n}(\cos \Theta)}{d \cos \Theta}
$$




$$
\tau_{n}(\cos \Theta)=\cos \Theta \pi_{n}(\cos \Theta)-\sin ^{2} \Theta \frac{d \pi_{n}(\cos \Theta)}{d \cos \Theta}
$$

The Mie theory is applied to calculate the radiative properties of single particles in this work. The calculation code is revised based on the code authored by Modest [20]. Test results calculated by the present code have very good consistency with those by Mahan [21], which shows the reliability of results calculated by both codes.

\subsection{Spectral Reflectivity and Emissivity of Coatings}

In order to fabricate a coating with uniform and required radiative properties, a large number of particles need to be dispersed uniformly in a matrix. In this work, the particle volume fraction is kept at a small value $(<5 \%)$, so only independent scattering is considered. If $N_{i}(i=1,2, \ldots, m)$ is the number of particles per unit volume with corresponding particle radius $r_{i},(i=1,2, \ldots, m)$, then the scattering coefficient $\sigma_{s} \lambda$, absorption coefficient $\kappa_{\lambda}$, and scattering phase function $\Phi_{T \lambda}(\Theta)$ of the composite can be calculated by

$$
\begin{gathered}
\sigma_{s \lambda}=\sum_{i=1}^{m} \pi r_{i}^{2} N_{i} Q_{s c a, i} \\
\kappa_{\lambda}=\sum_{i=1}^{m} \pi r_{i}^{2} N_{i} Q_{a b s, i} \\
\Phi_{T \lambda}(\Theta)=\frac{1}{\sigma_{s \lambda}} \sum_{i=1}^{m} \pi r_{i}^{2} N_{i} Q_{a b s, i} \Phi\left(r_{i}, \Theta\right)
\end{gathered}
$$

where $Q_{a b s, i}, Q_{s c a, i}$, and $\Phi\left(r_{i}, \Theta\right)$ are calculated from Eqs. (1)-(3) and with the correlation $Q_{e x t, i}=Q_{a b s, i}$ $+Q_{s c a, i}$. For coatings with uniform size particles, $m$ is equal to one. As shown in Fig. 2, particles with parallel or perpendicular polarization have different complex refractive index. These two types of 
particles are assumed to have the same number in the coating. Then, the average scattering and absorption coefficient, scattering phase function can be calculated by Eqs. (11)-(13).

After the effective radiative properties of the coating are obtained, the calculation model of the spectral reflectivity and emissivity of the coating can be simplified as shown in Fig. 3. The particle embedded coating is considered as a homogeneous medium with specified scattering coefficient $\sigma_{s} \lambda$, absorption coefficient $\kappa_{\lambda}$, and scattering phase function $\Phi_{T \lambda}(\Theta)$. The carbon black layer at the bottom is considered as the black boundary of the top $\mathrm{TiO}_{2}$ layer.

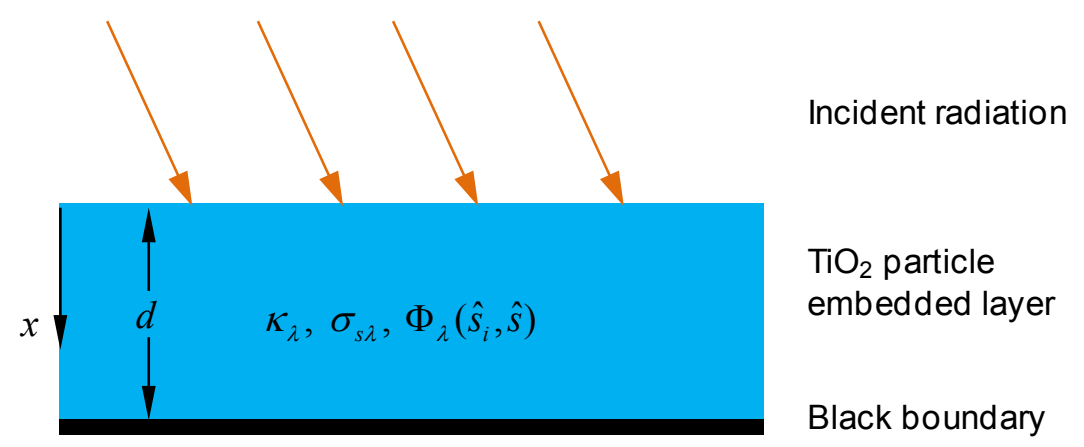

Figure 3 Calculation model of the RTE

After solving the RTE [20],

$$
\frac{d I_{\lambda}}{d s}=\kappa_{\lambda} I_{b \lambda}-\left(\kappa_{\lambda}+\sigma_{s \lambda}\right) I_{\lambda}+\frac{\sigma_{s \lambda}}{4 \pi} \int_{4 \pi} I_{\lambda}\left(\hat{s}_{i}\right) \Phi_{\lambda}\left(\hat{s}_{i}, \hat{s}\right) d \Omega_{i}
$$

with the boundary condition (when $x=d, \varepsilon=1.0$ ), the spectral reflectivity $\left(\rho_{\lambda}\right)$ of the double-layer coating is obtained. The spectral emissivity $\left(\varepsilon_{\lambda}\right)$ is equal to the spectral absorptivity according to Kirchhoff's law, which is calculated by $1-\rho_{\lambda}$ since there is no transmission because of the black boundary at the bottom. In this work the scattering phase function of the composite calculated by the Mie theory is complicated. Monte Carlo method can handle it without any approximation and is used here. 10,000 energy bundles are used for each case calculation and energy bundles are traced based on 
the path-length method used in Distributions of Ratios of Energy Scattered Or Reflected (DRESOR) method [22-23].

\subsection{Radiative Cooling Power of Coatings}

Consider a surface coated with the coating at temperature $T$, the net cooling power $P_{n e t}$ of the coating with area $A$ is given by [9]

$$
P_{n e t}(T)=P_{r a d}(T)-P_{a t m}\left(T_{a m b}\right)-P_{\text {sun }}-P_{c o n d+c o n v}
$$

where $T_{a m b}$ is the ambient temperature. $P_{\text {rad }}$ is the power emitted by the coating, $P_{a t m}$ is the absorbed power from the atmosphere radiation, $P_{\text {sun }}$ is the absorbed solar power, $P_{\text {cond }+ \text { conv }}$ is the absorbed power due to conduction and convection. They can be calculated by [9]

$$
\begin{gathered}
P_{r a d}(T)=A \int_{2 \pi} d \Omega \cos \theta \int_{0}^{\infty} d \lambda I_{B}(T, \lambda) \varepsilon(\lambda, \theta) \\
P_{a t m}\left(T_{a m b}\right)=A \int_{2 \pi} d \Omega \cos \theta \int_{0}^{\infty} d \lambda I_{B}\left(T_{a m b}, \lambda\right) \varepsilon(\lambda, \theta) \varepsilon_{a t m}(\lambda, \theta) \\
P_{s u n}=A \int_{0}^{\infty} d \lambda \alpha(\lambda, 0) I_{A M 1.5}(\lambda) \\
P_{\text {cond }+ \text { conv }}\left(T, T_{a m b}\right)=A h_{c}\left(T_{a m b}-T\right)
\end{gathered}
$$

where $I_{B}(T, \lambda)$ is the blackbody spectral intensity, $\varepsilon(\lambda, \theta)$ is the directional emissivity of the coating at wavelength $\lambda, \alpha(\lambda, 0)$ is the normal absorptivity of the coating at wavelength $\lambda, \varepsilon_{a t m}(\lambda, \theta)=1-t(\lambda)^{1 / \cos \theta}$ is the directional emissivity of the atmosphere, where $t(\lambda)$ is the atmospheric transmittance in the zenith direction [24]. The atmospheric transmittance varies with the column water vapor and air mass value. The column water vapor is assumed to be $1.0 \mathrm{~mm}$ and the air mass is 1.5 in the following calculations. $I_{A M 1.5}(\lambda)$ is the solar illumination with direct normal irradiance of $900 \mathrm{~W} / \mathrm{m}^{2}[25] . h_{c}$ is the combined conduction and convection heat transfer coefficient. In Eq. (18) it is assumed that the coating is facing 
the sun, so the absorptivity is equal to the emissivity in normal direction of the coating according to Kirchhoff's law.

\section{RESULTS AND DISCUSSION}

\subsection{Ideal Radiative Coolers}

The ideal radiative cooler is considered as the cooler with the ideal selective radiative properties, which can achieve the maximum net cooling power under specified surrounding conditions. As indicated in Eq. (15), the net cooling power of a radiative cooler is determined by four terms. The last term of the absorbed power due to conduction and convection is not affected by the cooler's radiative properties but the surrounding conditions, so it's not considered here to determine the ideal selective radiative properties of the cooler. Apparently, the ideal cooler needs to be an ideal emitter in the "sky window" and also an ideal reflector in the solar spectrum. Other than these two spectral ranges (0.3-2.5 and 8-13 $\mu \mathrm{m})$, the radiative property variation may have two opposite influences on the cooling power. For example, when the radiative cooler's temperature is lower than the atmosphere temperature, the increase of the surface emissivity will increase the cooler's emission power, at the same time, the power absorbed from the atmosphere also increases.

Figure 4(a) shows two ideal emitters which both have ideal radiative properties in the solar spectrum and the "sky window". However, outside of these two spectral ranges they have completely different properties, one with unity emissivity and the other with zero emissivity. The ambient 
temperature is assumed to be $300 \mathrm{~K}$. The cooling powers of these two emitters are shown in Fig. 4(b). According to the figure, emitter 1 can achieve a lower equilibrium temperature (the cooling power is equal to zero) than emitter 2, since it doesn't absorb heat from the atmosphere when its temperature is lower than the ambient. On the other hand, emitter 2 can generate more cooling power than emitter 1 if the surface temperature is higher than $280 \mathrm{~K}$. As discussed before, when the surface temperature is lower than the ambient temperature, the emissivity increase outside of the spectral ranges of the solar spectrum and the "sky window" will enhance the emission from the surface and the absorption from the atmosphere at the same time. When the surface temperature is higher than a specific value $(280 \mathrm{~K}$ in this case), the increase in emission is dominant over the increase in absorption, leading to a better cooling performance of emitter 2 .

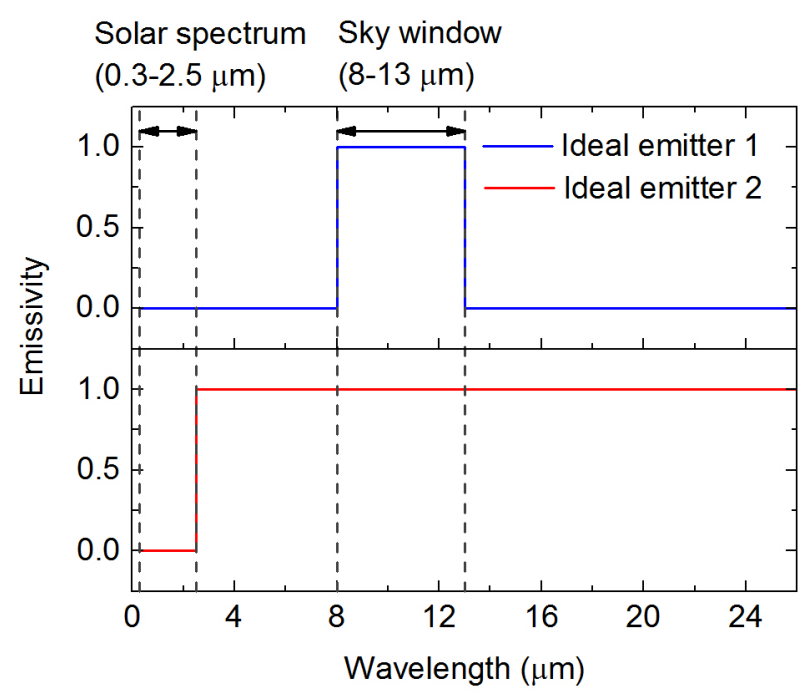

(a)

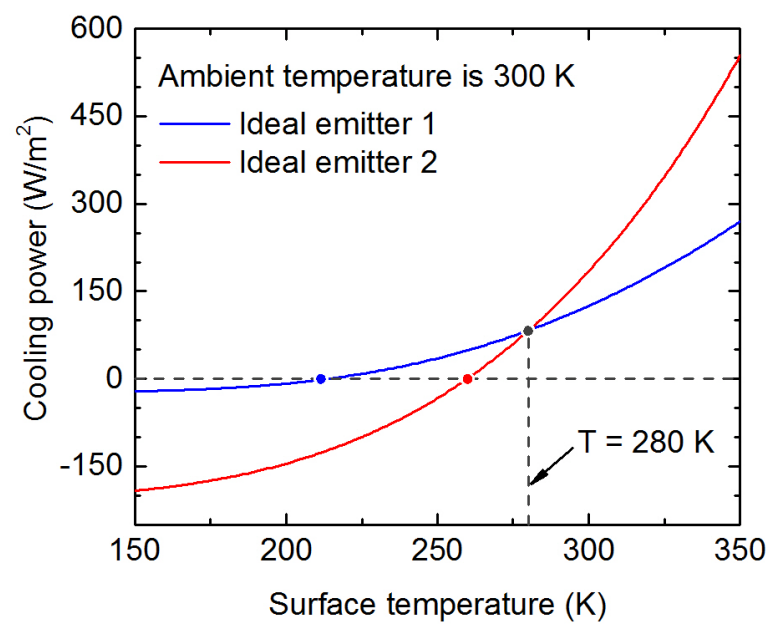

(b)

Figure 4 Cooling performance of two ideal emitters (a) spectral emissivity (b) cooling power

In a word, the ideal radiative cooler must be an ideal reflector in the solar spectrum range and an ideal emitter in the "sky window". Outside of these two spectral ranges, depending on the cooling 
purpose (low equilibrium temperature or high cooling power) and operation temperature, the requirement of the spectral radiative properties may be different. In the following discussion, we will mainly focus on the radiative properties in the solar spectrum and the "sky window".

\subsection{Radiative Properties of Particle Embedded Coatings}

In order to investigate the effect of particle sizes, four coatings embedded with titanium dioxide particles with radius $r=0.1,0.2,0.3$, and $0.4 \mu \mathrm{m}$ respectively are considered. The particle volume fractions of the coatings are kept the same at $4 \%$. The thickness of the coatings is $500 \mu \mathrm{m}$. The scattering coefficients of these coatings are shown in Fig. 5(a). Each curve shows a scattering peak which shifts to longer wavelength as the particle sizes increase. Also, as the particle size increases, the scattering coefficient has a weaker dependence on the wavelength. The spectral reflectivity is calculated under the normal irradiation in Fig. 5(b). It can be seen that as the particle size increases, the reflectivity in long wavelength increases and the curve becomes relatively flat in the whole solar spectrum, but the reflectivity in the visible band becomes smaller. Therefore, changing the particle size yields two opposite effects for daytime radiative cooling performance, and the particle size optimization will be accomplished in the next section. 


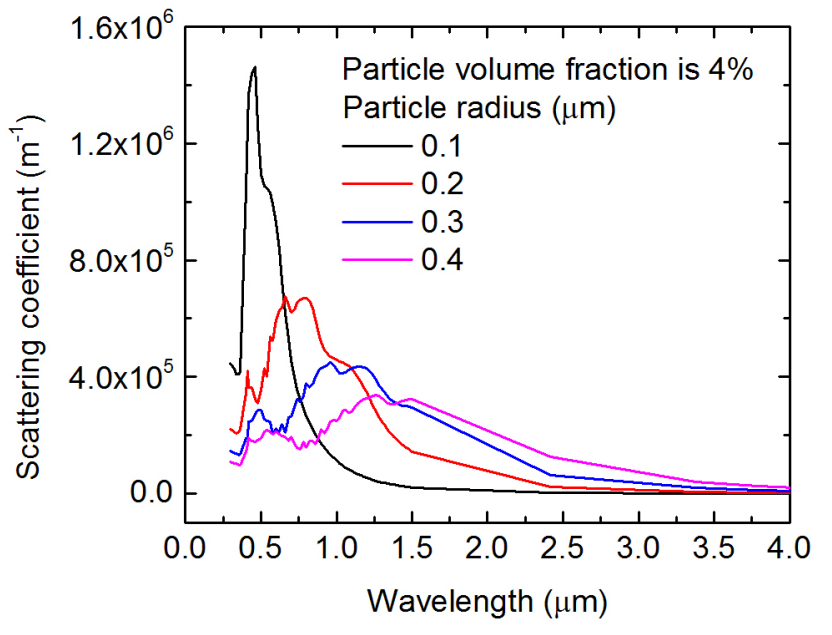

(a)

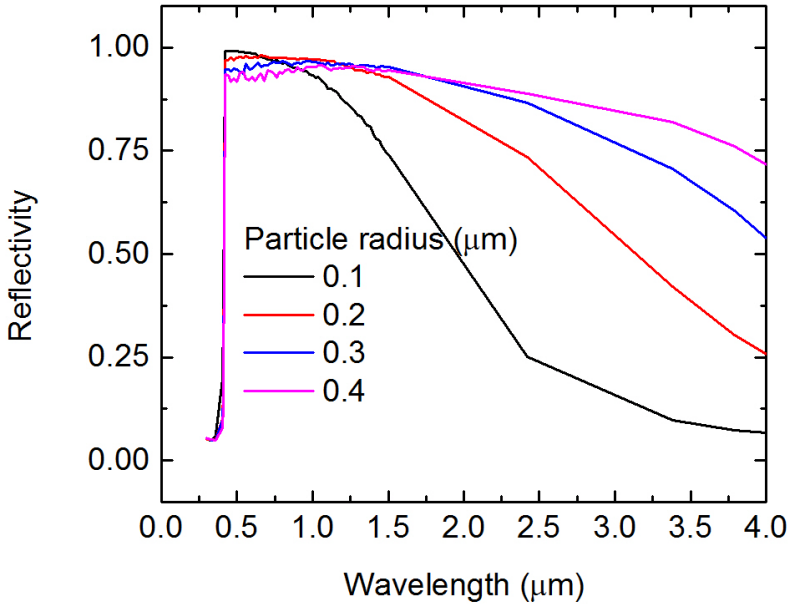

(b)

Figure 5 Radiative properties of particle embedded coatings (a) scattering coefficient (b) reflectivity

The reflectivity in the "sky window" for all four coatings is very small (not shown in Fig. 5) since the particles are too small to be "seen" by the long wavelength in the "sky window". This leads to a high emissivity of the coating in this spectral range and benefits for emitting heat to the deep space.

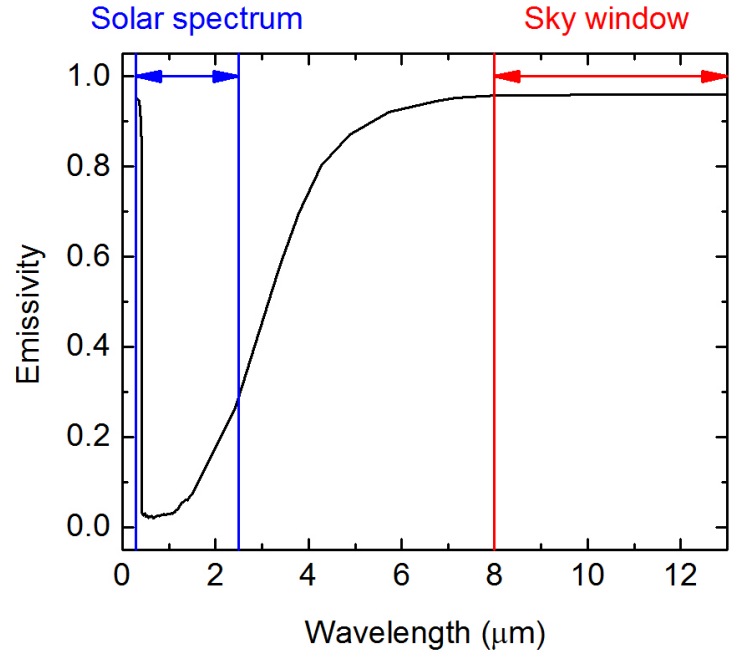

(a)

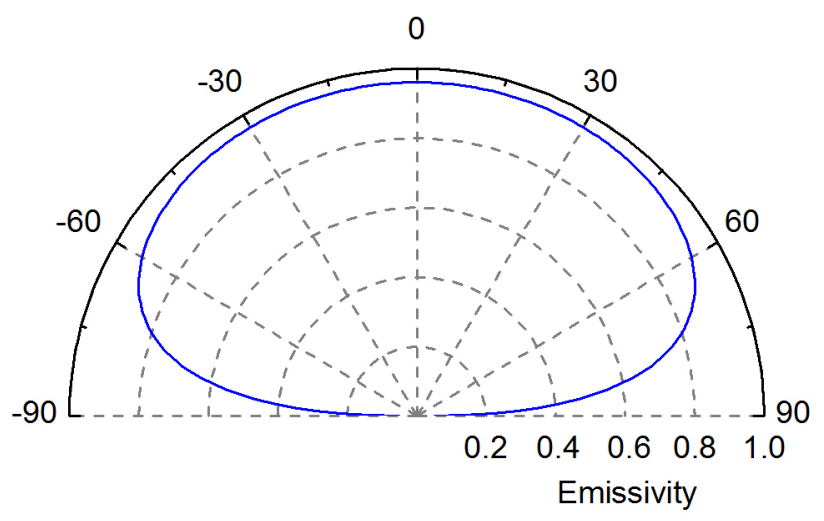

(b)

Figure 6 Emissivity of the coating with particle radius $r=0.2 \mu \mathrm{m}$ (a) normal direction (b) average 
emissivity in the "sky window" $(8-13 \mu \mathrm{m})$

The spectral and directional emissivity of the double-layer coating is shown in Fig. 6. Since all the coatings with different particle sizes have similar trend, only the coating embedded with particles of $0.2 \mu \mathrm{m}$ in radius is shown here for brevity. The coating thickness is $500 \mu \mathrm{m}$ and the volume fraction of particles is $4 \%$. The carbon black layer is considered as a black substrate. The spectral emissivity in the normal direction is shown in Fig. 6(a). Because there is no transmission through the coating, the sum of the spectral reflectivity $\rho$ and absorptivity $\alpha$ is equal to unity. According to Kirchhoff's law the emissivity $\varepsilon$ equals to the absorptivity $\alpha$, then we have $\rho=1-\varepsilon$. From the figure, the coating has low emissivity in the solar spectrum, especially in the short wavelength range in which the sun irradiates strongly. About $91 \%$ of the solar energy is reflected by the coating, which is very important for the daytime cooling. At the same time, the coating has very high emissivity (more than 0.95 ) in the "sky window" and can maximally emit heat out to the deep space.

Figure 6(b) shows the directional average emissivity in the "sky window". The emissivity is more than 0.90 in most directions except when the angle is larger than 60 degrees. This is a good feature to maximize the emission power $\left(P_{r a d}\right)$ according to Eq. (16). The results in Fig. 6 show that the coating has the desirable properties for daytime radiative cooling.

\subsection{Cooling Performance of Particle Embedded Coatings}

The cooling performance of coatings embedded with different size particles is shown in Fig. 7. The power emitted by the coating $\left(P_{r a d}\right)$, the absorbed power from the atmosphere radiation $\left(P_{a t m}\right)$, the 
absorbed solar power $\left(P_{\text {sun }}\right)$, and the net cooling power $\left(P_{n e t}\right)$ are plotted in this figure. The coatings are the same as those shown in Fig. 5. The ambient temperature is $300 \mathrm{~K}$ and the coating is assumed to be held at the same temperature as the ambient. Therefore, there is no net conduction or convection. As shown in the figure, the emitted power by the surface $\left(P_{r a d}\right)$ and the absorbed power from the atmosphere $\left(P_{a t m}\right)$ do not change much as the particle size varies. This is because $P_{r a d}$ and $P_{a t m}$ are mainly influenced by the radiative properties in the infrared range $(\lambda>4.5 \mu \mathrm{m})$, and different size of the particles does not cause much difference of the radiative properties at long wavelength. On the other hand, the absorbed power from the solar $\left(P_{\text {sun }}\right)$ is mainly influenced by the radiative properties in the solar spectrum $(0.3-2.5 \mu \mathrm{m})$, which varies significantly with different particle sizes. The coating with particle radius of $0.2 \mu \mathrm{m}$ absorbs the least solar energy and has the best cooling performance among the considered coatings. The radius of $0.2 \mu \mathrm{m}$ is considered as the optimized size of the titanium dioxide particles for the daytime radiative cooling, and the following discussion will focus on the coating embedded with these specific particles.

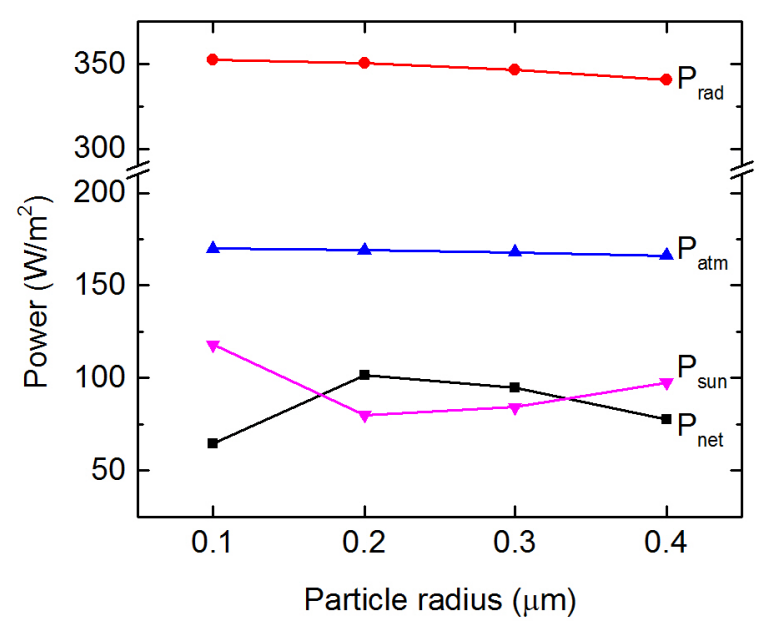

Figure 7 Net cooling power $\left(P_{n e t}\right)$ and its components $\left(P_{r a d}, P_{a t m}, P_{\text {sun }}\right)$ for coatings embedded with 


\section{different size particles}

The cooling performance of the coating is further investigated by taking into consideration of conduction and convection heat exchange. Three different combined coefficients of conduction and convection $h_{c}=0,6,12 \mathrm{~W} /\left(\mathrm{m}^{2} \mathrm{~K}\right)$ are considered, in which $h_{c}=0$ means the conduction and convection is negligible, $h_{c}=6,12 \mathrm{~W} /\left(\mathrm{m}^{2} \mathrm{~K}\right)$ represents the cases that the wind speed is about $1 \mathrm{~m} / \mathrm{s}$ and $3 \mathrm{~m} / \mathrm{s}$, respectively [26], and assume the coating is coated on a structure such as the polystyrene and the conduction coefficient is negligible compared to the convection coefficient. The ambient temperature is $300 \mathrm{~K}$.

As shown in Fig. 8(a), this coating has the potential to achieve a net cooling power of $100 \mathrm{~W} / \mathrm{m}^{2}$ at ambient temperature under direct solar irradiation. At nighttime the cooling power is more than 180 $\mathrm{W} / \mathrm{m}^{2}$ at the ambient temperature as shown in Fig. $8(\mathrm{~b})$. The coating can achieve an equilibrium temperature of $278 \mathrm{~K}$ and $255 \mathrm{~K}$ at daytime and nighttime, respectively. Consider a practical case, if the wind speed above the coating is around $3 \mathrm{~m} / \mathrm{s}$, which corresponds to the case that $h_{c}$ is equal to 12 $\mathrm{W} /\left(\mathrm{m}^{2} \mathrm{~K}\right)$, the cooling power at the ambient temperature is not affected. The cooling power at the temperature higher than the ambient temperature is even larger than the case of $h_{c}=0$ thanks to the cooling effect by the convection. When the surface temperature is lower than the ambient temperature, the convection results in heating, and the coating can still achieve cooling effect with the equilibrium temperature of $294 \mathrm{~K}$ at daytime and $289 \mathrm{~K}$ at nighttime. 


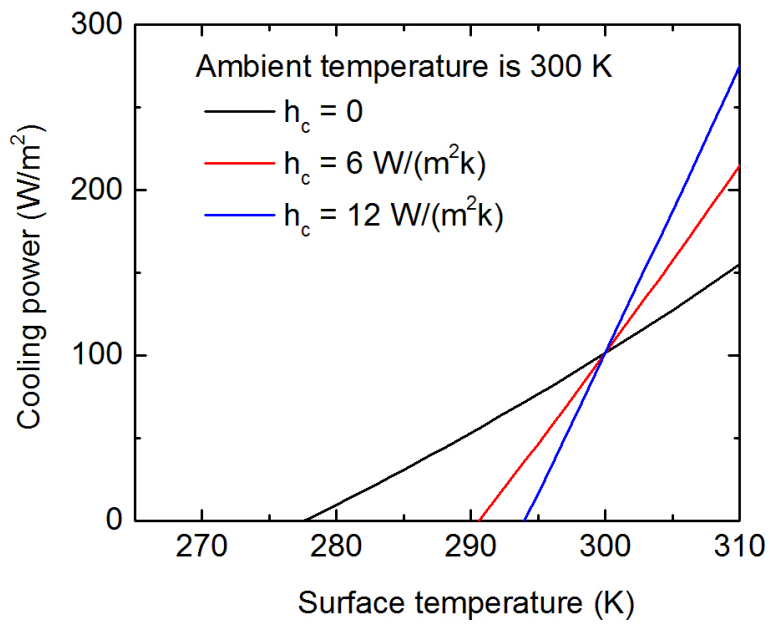

(a)

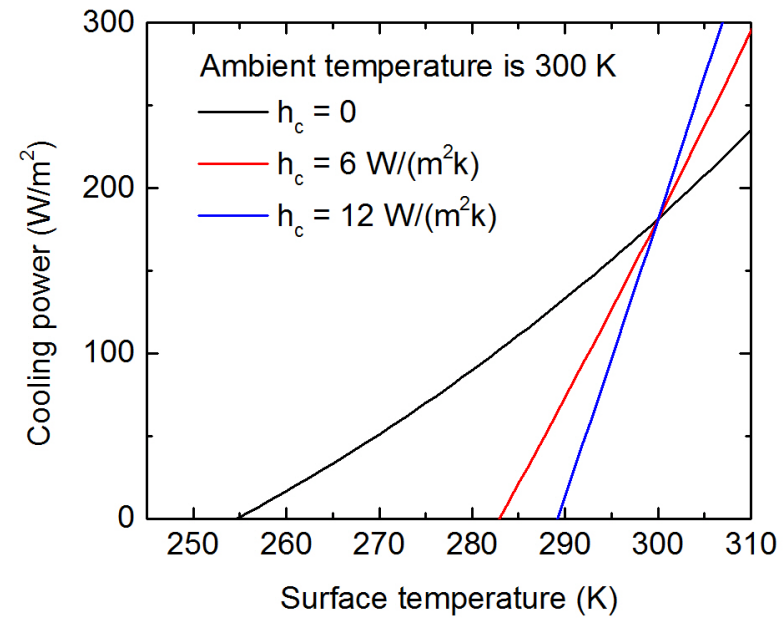

(b)

Figure 8 Cooling power of the coating with particle radius $r=0.2 \mu \mathrm{m}$ (a) at daytime (b) at nighttime

\section{CONCLUSIONS}

In this work we have proposed a cost-effective double-layer coating embedded with particles for the first time for both nighttime and daytime radiative cooling. The two layers are acrylic resin embedded with titanium dioxide and carbon black particles. First, the radiative properties of ideal radiative coolers are analyzed. The ideal radiative cooler must have high reflectivity in the solar spectrum and high emissivity in the atmospheric transparency window. Outside of these two spectral ranges, depending on the cooling purpose and operation temperature, the requirement of the spectral radiative properties is different. Then, the cooling performance of the designed double-layer coatings is investigated. The spectral reflectivity and emissivity of the coating are calculated by the Mie theory and solving the Radiative Transfer Equation. Different sizes of the titanium dioxide particles are 
examined for the cooling purpose. Results show that the coating embedded with particles of $0.2 \mu \mathrm{m}$ in radius has the best cooling performance. More than $90 \%$ of the solar irradiation can be reflected, and the average emissivity in the atmospheric transparency window is larger than 0.9 in most directions. Net cooling power over $100 \mathrm{~W} / \mathrm{m}^{2}$ at daytime and over $180 \mathrm{~W} / \mathrm{m}^{2}$ at nighttime is achieved at ambient temperature. The lower achieved equilibrium temperature than the ambient temperature shows the cooling effect persists even taking consideration of significant conduction and convection heat exchange.

\section{ACKNOWLEDGEMENTS}

ZH acknowledges partial support by the National Natural Science Foundation of China (No. 51106058) and the International Postdoctoral Exchange Fellowship Program (No. 20130022). XR acknowledges the partial support of the National Science Foundation (Award No. 1150948).

\section{REFERENCES}

[1] E.M. Lushiku, A. Hjortsberg, C.G. Granqvist, Radiative cooling with selectively infrared emitting ammonia gas, Journal of Applied Physics, 53(8) (1982) 5526-5530.

[2] E.M. Lushiku, C.-G. Granqvist, Radiative cooling with selectively infrared-emitting gases, Applied Optics, 23(11) (1984) 1835-1843.

[3] S. Catalanotti, V. Cuomo, G. Piro, D. Ruggi, V. Silvestrini, G. Troise, The radiative cooling of 
selective surfaces, Solar Energy, 17(2) (1975) 83-89.

[4] C.G. Granqvist, A. Hjortsberg, Radiative cooling to low temperatures: General considerations and application to selectively emitting SiO films, Journal of Applied Physics, 52(6) (1981) $4205-4220$.

[5] D.M. Diatezua, P.A. Thiry, A. Dereux, R. Caudano, Silicon oxynitride multilayers as spectrally selective material for passive radiative cooling applications, Solar Energy Materials and Solar Cells, 40(3) (1996) 253-259.

[6] L. Zhu, A. Raman, S. Fan, Color-preserving daytime radiative cooling, Applied Physics Letters, $103(22)(2013) 223902$.

[7] L. Zhu, A. Raman, K.X. Wang, M.A. Anoma, S. Fan, Radiative cooling of solar cells, Optica, 1(1) (2014) 32-38.

[8] E. Rephaeli, A. Raman, S. Fan, Ultrabroadband Photonic Structures To Achieve High-Performance Daytime Radiative Cooling, Nano Letters, 13(4) (2013) 1457-1461.

[9] A.P. Raman, M.A. Anoma, L. Zhu, E. Rephaeli, S. Fan, Passive radiative cooling below ambient air temperature under direct sunlight, Nature, 515(7528) (2014) 540-544.

[10]A.W. Harrison, M.R. Walton, Radiative cooling of TiO2 white paint, Solar Energy, 20(2) (1978) $185-188$.

[11]A. Andretta, B. Bartoli, B. Coluzzi, V. Cuomo, Selective Surfaces for Natural Cooling Devices, J. Phys. Colloques, 42(C1) (1981) C1-423-C1-430.

[12]T.M. Nilsson, G.A. Niklasson, Optimization of optical properties of pigmented foils for radiative cooling applications: model calculations, in: Proc. SPIE 1536, Optical Materials Technology for 
Energy Efficiency and Solar Energy Conversion X, San Diego, CA, 1991, pp. 169-182.

[13]T.M.J. Nilsson, G.A. Niklasson, C.G. Granqvist, A solar reflecting material for radiative cooling applications: ZnS pigmented polyethylene, Solar Energy Materials and Solar Cells, 28(2) (1992) 175-193.

[14]T.M.J. Nilsson, G.A. Niklasson, Radiative cooling during the day: simulations and experiments on pigmented polyethylene cover foils, Solar Energy Materials and Solar Cells, 37(1) (1995) 93-118.

[15]A.R. Gentle, G.B. Smith, Radiative Heat Pumping from the Earth Using Surface Phonon Resonant Nanoparticles, Nano Letters, 10(2) (2010) 373-379.

[16]H. Gonome, M. Baneshi, J. Okajima, A. Komiya, S. Maruyama, Controlling the radiative properties of cool black-color coatings pigmented with $\mathrm{CuO}$ submicron particles, Journal of Quantitative Spectroscopy and Radiative Transfer, 132(0) (2014) 90-98.

[17]H. Gonome, M. Baneshi, J. Okajima, A. Komiya, N. Yamada, S. Maruyama, Control of thermal barrier performance by optimized nanoparticle size and experimental evaluation using a solar simulator, Journal of Quantitative Spectroscopy and Radiative Transfer, 149(0) (2014) 81-89.

[18]E.D. Palik, Handbook of Optical Constants of Solids, in, Academic Press, San Diego, 1985.

[19]J.R. Howell, M.P. Menguc, R. Siegel, Thermal Radiation Heat Transfer, 5 th ed., CRC Press, 2011, P11.

[20]M.F. Modest, Radiative heat transfer, second ed., Academic Press, San Diego, 2003, P391.

[21]J.R. Mahan, Radiation Heat Transfer: A Statistical Approach, Wiley, 2002.

[22]H.-C. Zhou, Q. Cheng, Z.-F. Huang, C. He, The influence of anisotropic scattering on the radiative 
intensity in a gray, plane-parallel medium calculated by the DRESOR method, Journal of Quantitative Spectroscopy and Radiative Transfer, 104(1) (2007) 99-115.

[23]Z. Huang, H. Zhou, Q. Cheng, P.-f. Hsu, Solution of radiative intensity with high directional resolution in three-dimensional rectangular enclosures by DRESOR method, International Journal of Heat and Mass Transfer, 60(0) (2013) 81-87.

[24]IR Transmission Spectra, Gemini Observatory. http://www.gemini.edu/?q=node/10789 (accessed May 11, 2015)

[25] Standard Tables for Reference Solar Spectral Irradiances: Direct Normal and Hemispherical on $37^{\circ}$ Tilted Surface, ASTM International, Designation: G173 - 03 (Reapproved 2012).

[26]J.A. Duffie, W.A. Beckman, Solar Engineering of Thermal Processes, 4th ed., John Wiley \& Sons, Inc., Hoboken, New Jersey, 2013, P164. 